\title{
A Scalable High-Frequency Noise Model for Bipolar Transistors with Application to Optimal Transistor Sizing for Low-Noise Amplifier Design
}

\author{
Sorin P. Voinigescu, Member, IEEE, Michael C. Maliepaard, Member, IEEE, Jonathan L. Showell, Member, IEEE, \\ Greg E. Babcock, David Marchesan, Member, IEEE, Michael Schroter, Member, IEEE, \\ Peter Schvan, Member, IEEE, and David L. Harame, Member, IEEE
}

\begin{abstract}
Fully scalable, analytical HF noise parameter equations for bipolar transistors are presented and experimentally tested on high-speed $\mathrm{Si}$ and SiGe technologies. A technique for extracting the complete set of transistor noise parameters from $Y$ parameter measurements only is developed and verified. Finally, the noise equations are coupled with scalable variants of the HICUM and SPICE-Gummel-Poon models and are employed in the design of tuned low noise amplifiers (LNA's) in the 1.9-, 2.4-, and 5.8-GHz bands.
\end{abstract}

Index Terms-Bipolar transistor, compact modeling, low noise, low-noise amplifier, low-noise transistor design, noise figure, noise matching, noise measurements, radio-frequency integrated circuit design, SiGe heterojunction bipolar transistor.

\section{INTRODUCTION}

$\mathbf{T}$ HE recent boom in wireless consumer applications has emphasized the requirement for low-cost, highly integrated RF parts. Steady improvement in transistor performance and desire for higher level of integration have led to the increased application of silicon technology. Since substrate and interconnect losses are significantly higher in $\mathrm{Si}$ than in GaAs, Si RF circuit design should target the optimization of the size of transistors in order to simplify matching, rather than design the matching circuit around a given transistor. Such an approach requires a physically based, scalable compact model for bipolar transistors [1], as well as accurate, closed-form noise parameter equations, suitable for circuit design.

In the first part, expressions for the four noise parameters of a bipolar transistor are derived. The accuracy of these equations is investigated using SPICE-Gummel-Poon (SGP) modeled and measured transistor noise data. Next, a technique is presented for extracting the complete set of noise parameters from measured $Y$ parameters only. It avoids the inaccuracy of on-wafer noise parameter measurements which is especially severe for small geometry devices or devices biased in the sub-

Manuscript received February 3, 1997; revised April 25, 1997.

S. P. Voinigescu, M. C. Maliepaard, J. L. Showell, G. E. Babcock, D. Marchesan, and P. Schvan are with Nortel Technology, Northern Telecom, Ottawa, ON K1Y 4H7, Canada.

M. Schroter was with Nortel Technology, Northern Telecom, Ottawa, ON K1Y 4H7, Canada. He is now with Rockwell Semiconductor Systems, Newport Beach, CA 92660 USA.

D. L. Harame was with IBM Microelectronic Division, Hopewell Junction, NY 12533-6531 USA. He is now with IBM, Essex Junction, VT 05452 USA. Publisher Item Identifier S 0018-9200(97)05770-3.
$\mathrm{mA}$ range. Finally, scalable variants of the SGP and HICUM models [2], in conjunction with the new noise parameter equations, are applied in the design of tuned low-noise amplifiers (LNA's) in the 2-6 GHz band.

\section{NoISE PARAMETER EQUATIONS AND MEASUREMENTS}

\section{A. Theoretical Background}

Equations (1)-(3) define the noise resistance $R_{n}$, optimum source admittance $Y_{\text {sop }}$, and minimum noise figure $F_{\mathrm{MIN}}$ of a two-port with respect to its noise correlation matrix entries $C_{A 11}, C_{A 12}$, and $C_{A 22}$ [3]-[4]. The latter, in turn, are expressed in (4)-(6) as functions of the input-referred noise voltage $v_{n}^{2}$ and noise current $i_{n}^{2}$ sources of the two-port, as well as of the $Y$ parameters of the noise-free two-port

$$
\begin{aligned}
R_{n}= & C_{A 11} \\
Y_{\mathrm{sop}}= & G_{\mathrm{SOP}}+j B_{\mathrm{SOP}}=\sqrt{\frac{C_{A 22}}{C_{A 11}}-\left(\frac{\operatorname{Im}\left\{C_{A 12}\right\}}{C_{A 11}}\right)^{2}} \\
& +j \frac{\operatorname{Im}\left\{C_{A 12}\right\}}{C_{A 11}} \\
F_{\mathrm{MIN}}= & 1+2\left(\operatorname{Re}\left\{C_{A 12}\right\}+C_{A 11} G_{\mathrm{sop}}\right) \\
C_{A 11}= & \frac{\left\langle v_{n}^{2}\right\rangle}{4 k T \Delta f}=\frac{\left\langle i_{c}^{2}\right\rangle}{4 k T \Delta f\left|Y_{21}\right|^{2}}+\left(r_{E}+r_{B}\right) \\
C_{A 21}= & C_{A 12}^{*}=\frac{\left\langle v_{n}^{*} i_{n}\right\rangle}{4 k T \Delta f}=\frac{Y_{11} \cdot\left\langle i_{c}^{2}\right\rangle}{4 k T \Delta f\left|Y_{21}\right|^{2}} \\
C_{A 22}= & \frac{\left\langle i_{n}^{2}\right\rangle}{4 k T \Delta f}=\frac{\left|Y_{11}\right|^{2} \cdot\left\langle i_{c}^{2}\right\rangle}{4 k T \Delta f\left|Y_{21}\right|^{2}}+\frac{\left\langle i_{b}^{2}\right\rangle}{4 k T \Delta f}
\end{aligned}
$$

Equation (3) is a recast of the original formula in [3]. Fig. 1 illustrates the simplified small-signal and noise equivalent circuit and the two-step approach used in the derivation of the noise parameters in the case of a bipolar transistor. The noise current sources $i_{B}^{2}, i_{C}^{2}$ represent the internal shot noise sources of the bipolar transistor. After substituting (4)-(6) into (1)-(3), (7)-(9) are obtained which describe the bipolar transistor noise parameters as functions of the internal shot noise current sources, transistor $Y$ parameters, series emitter resistance $r_{E}$, and total base resistance $r_{B}$ in a manner similar to that employed for GaAs MESFET's [5]. The bias current 


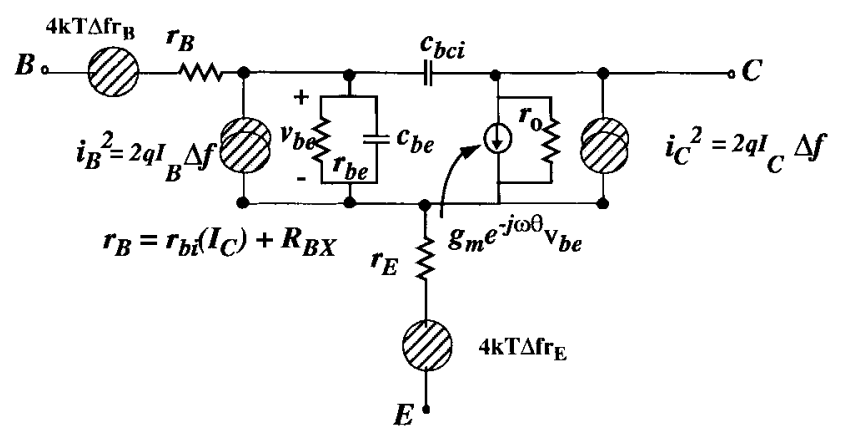

(a)

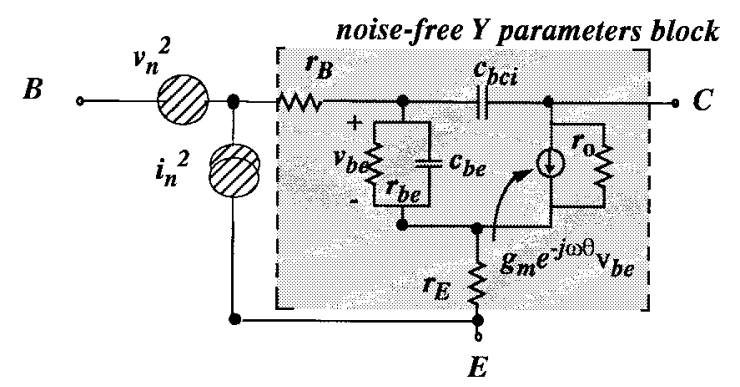

(b)

Fig. 1. (a) Small signal and noise equivalent circuit used in the bipolar transistor noise parameter derivation. It features the uncorrelated shot-noise currents $i_{B}^{2}$ and $i_{C}^{2}$. (b) The equivalent input-referred noise model with correlated noise sources $i_{n}^{2}, v_{n}^{2}$, and noise-free $Y$ parameters block.

dependence of the noise parameters appears in explicit form via the $I_{B}$ and $I_{C}$ terms, and also implicitly in $r_{B}$ and in the $Y$ parameters. $V_{T}$ represents the thermal voltage. In the derivation of (7)-(9) it is assumed that the base and collector noise currents are uncorrelated. This is equivalent to neglecting the imaginary part of the transconductance $g_{m} e^{-j \omega \theta}$, a reasonable simplification up to frequencies approaching $f_{T} / 2$ [6]. $\theta$ is the transconductance delay, typically $40-60 \%$ of the transit time [7]. Despite this assumption, the input-referred noise sources $v_{n}^{2}$ and $i_{n}^{2}$ remain correlated and are modeled accordingly [see (8) and (9) at the bottom of the page]

$$
R_{n}=\frac{I_{C}}{2 V_{T}\left|Y_{21}\right|^{2}}+\left(r_{E}+r_{B}\right)
$$

Similarly, Si MOSFET noise parameters can be obtained by making the following substitutions in (7)-(9):

$$
\left(\frac{I_{C}}{2 V_{T}} \rightarrow \frac{2}{3} g_{m}\right) ;\left(I_{B} \rightarrow 0\right) ;\left(r_{E}+r_{B}\right) \rightarrow\left(R_{S}+R_{G}\right) .
$$

For the bias currents and frequency range used in wireless design, the bipolar transistor noise parameter equations can be recast in easy-to-interpret formats (10)-(13). These can be employed to tailor the device size-typically only the emitter length $l_{E}$-to achieve optimal low-noise matching at the desired frequency and input impedance. As indicated by (10) and (11), the noise resistance and optimum noise admittance scale as $l_{E}^{-1}$ and $l_{E}$, respectively. Alternatively, the real part of the optimum noise impedance $R_{\mathrm{SOP}}$, given by (12), scales as $l_{E}^{-1}$ and decreases with increasing frequency, $f$

$$
\begin{aligned}
& R_{n} \cong \frac{n^{2} V_{T}}{2 I_{C}}+\left(r_{E}+r_{B}\right) \sim l_{E}^{-1} \\
& Y_{\text {sop }} \cong \frac{f}{f_{T} R_{n}} \\
& \times\left\{\sqrt{\frac{I_{C}}{2 V_{T}}\left(r_{E}+r_{B}\right)\left(1+\frac{f_{T}^{2}}{\beta_{0} f^{2}}\right)+\frac{n^{2} f_{T}^{2}}{4 \beta_{0} f^{2}}}-j \frac{n}{2}\right\} \\
& \sim l_{E} \\
& R_{\mathrm{sop}} \cong \frac{R_{n} f_{T}}{f} \\
& \times \frac{\sqrt{\frac{I_{C}}{2 V_{T}}\left(r_{E}+r_{B}\right)\left(1+\frac{f_{T}^{2}}{\beta_{0} f^{2}}\right)+\frac{n^{2} f_{T}^{2}}{4 \beta_{0} f^{2}}}}{\frac{I_{C}}{2 V_{T}}\left(r_{E}+r_{B}\right)\left(1+\frac{f_{T}^{2}}{\beta_{0} f^{2}}\right)+\frac{n^{2}}{4}\left(1+\frac{f_{T}^{2}}{\beta_{0} f^{2}}\right)}=Z_{0}
\end{aligned}
$$

where $\beta_{0}$ is the dc current gain. $n$ is the collector current ideality factor, approximately equal to one, except under high current injection bias when its value can exceed 1.2. As long as the length-to-width ratio $\left(l_{E} / w_{E}\right)$ of the emitter stripe is larger than ten, $F_{\mathrm{MIN}}$ remains invariant to changes in emitter length and increases almost linearly with frequency

$$
\begin{aligned}
F_{\mathrm{MIN}} \cong & +\frac{n}{\beta_{0}}+\frac{f}{f_{T}} \\
& \times \sqrt{\frac{2 I_{C}}{V_{T}}\left(r_{E}+r_{B}\right)\left(1+\frac{f_{T}^{2}}{\beta_{0} f^{2}}\right)+\frac{n^{2} f_{T}^{2}}{\beta_{0} f^{2}}} .
\end{aligned}
$$

All noise parameters are nonlinear functions of emitter width $w_{E}$ via the $I_{C}\left(r_{E}+r_{B}\right)$ term.

\section{B. Experimental Validation}

In order to verify the noise parameter equations, automated noise parameter measurements were carried out in the 2-6 GHz range using an NP5 on-wafer measurement system from ATN Microwave Inc. Devices with variable widths, lengths, and with single- or multi-stripe geometries have been investigated in three technologies: 1) a single-poly BiCMOS

$$
\begin{aligned}
Y_{\text {sop }} & =\sqrt{\frac{I_{B}\left|Y_{21}\right|^{2}+I_{C}\left|Y_{11}\right|^{2}}{2 V_{T}\left|Y_{21}\right|^{2}\left(r_{E}+r_{B}\right)+I_{C}}-\left(\frac{I_{C} \operatorname{Im}\left\{Y_{11}\right\}}{2 V_{T}\left|Y_{21}\right|^{2}\left(r_{E}+r_{B}\right)+I_{C}}\right)^{2}}-j \frac{I_{C} \operatorname{Im}\left\{Y_{11}\right\}}{2 V_{T}\left|Y_{21}\right|^{2}\left(r_{E}+r_{B}\right)+I_{C}} \\
F_{\mathrm{MIN}} & =1+\frac{I_{C}}{V_{T}\left|Y_{21}\right|^{2}}\left(\operatorname{Re}\left\{Y_{11}\right\}+\sqrt{\left[1+\frac{2 V_{T}\left|Y_{21}\right|^{2}\left(r_{E}+r_{B}\right)}{I_{C}}\right]\left[\left|Y_{11}\right|^{2}+\frac{I_{B}\left|Y_{21}\right|^{2}}{I_{C}}\right]-\left(\operatorname{Im}\left\{Y_{11}\right\}\right)^{2}}\right)
\end{aligned}
$$




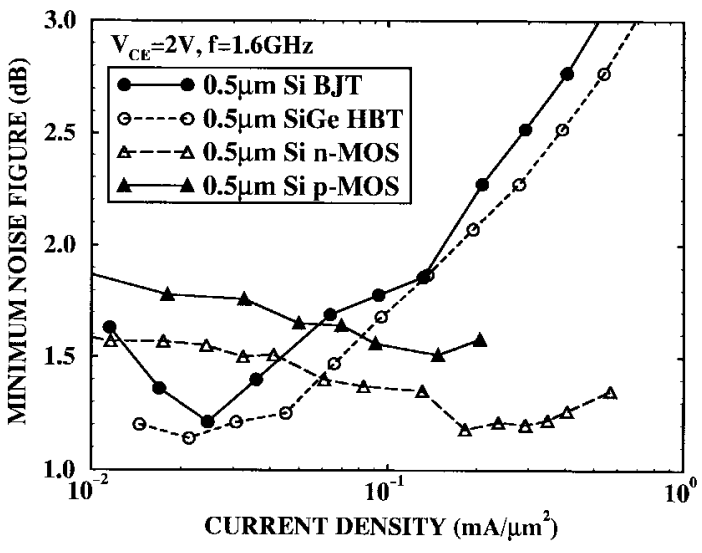

Fig. 2. ATN-NP5, on-wafer measured minimum noise figure as a function of drain/collector current density for state-of-the-art Si MOSFET's, Si BJT's, and SiGe HBT's with $0.5-\mu \mathrm{m}$ minimum feature size. All measurements were performed at $1.6 \mathrm{GHz}$. The unit gate finger width of the multifinger MOSFET's is $10 \mu \mathrm{m}$. The series and parallel parasitics associated with probing pads were not de-embedded. The drain current density in MOSFET's is obtained as $I_{D} /(W * L)$.

npn; 2) NT25, Northern Telecom's self-aligned, double-poly, implanted-base, Si npn, and 3) IBM's self-aligned, doublepoly SiGe heterojunction bipolar transistor (HBT) [8]. The peak cutoff frequencies at $V_{\mathrm{CE}}=2 \mathrm{~V}$ are 20, 26, and $45 \mathrm{GHz}$, respectively, while typical $f_{\mathrm{MAX}}$ values are 27 , 47 , and $65 \mathrm{GHz}$, respectively. In the case of the first two technologies, which do not feature trench isolation, $f_{\mathrm{MAX}}$ is a function of emitter length and decreases for shrinking emitter length-to-width ratios as a result of the lack of scaling in the collector-substrate capacitance.

Fig. 2 presents the measured minimum noise figure at $1.6 \mathrm{GHz}$ as a function of drain/collector current density for $0.5-\mu \mathrm{m}$ Si MOSFET's, Si bipolar junction transistors (BJT's), and SiGe HBT's. In the case of Si MOSFET's, dominated by thermal noise, minimum noise is achieved at large current densities, corresponding to the peak $f_{T}$ current density. Despite smaller $f_{T}$ values $(18-20 \mathrm{GHz})$ [9], n-MOSFET noise is as low as that of SiGe HBT's. However, in MOSFET's minimum noise can only be achieved by compromising lowpower consumption. In contrast, because of the interplay between thermal noise (from $r_{E}$ and $r_{B}$ ), prevailing at low current densities, and shot noise, dominating at high current densities, bipolar transistors show an optimum noise current density ten times smaller than the peak $f_{T}$ current density. Bipolar transistors have the benefit of concomitant low-noise and low-power operation. This advantage may diminish as MOSFET gate lengths continue to shrink [10]. Figs. 3 and 4 illustrate the measured emitter length and emitter width dependencies of the noise parameters of the NT25 Si BJT's. Both plots follow closely the predictions of the simplified noise parameter equations (10)-(13).

While noise parameters are available from the postprocessor of microwave circuit simulators such as HP-EESOF's LIBRA, only equivalent noise voltages and currents can be modeled directly using SPICE-like simulators. To circumvent this problem, (7)-(9) were included in an HSPICE deck and, in conjunction with the MEASURE statement, were employed

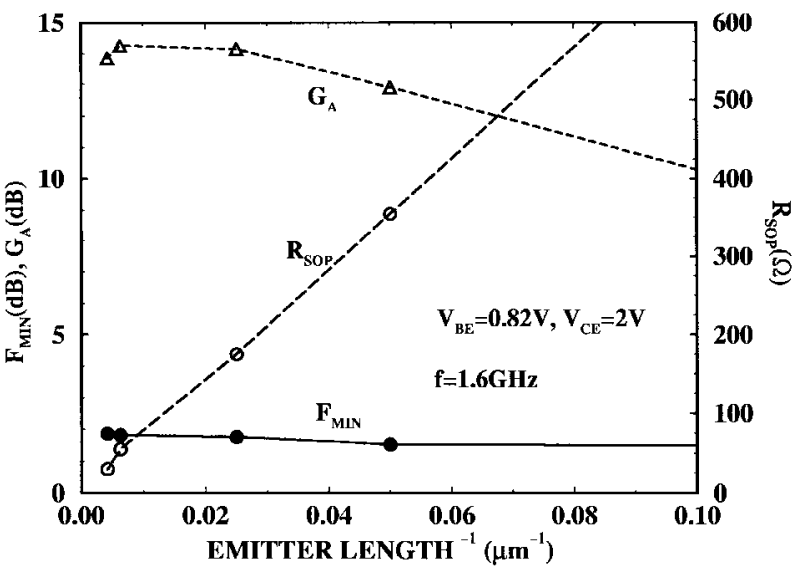

Fig. 3. ATN-NP5 on-wafer measured minimum noise figure $F_{\mathrm{MIN}}$, optimum noise resistance $R_{\mathrm{SOP}}$, and associated gain $G_{A}$, as functions of emitter length for NT25 Si BJT's with 0.5- $\mu \mathrm{m}$ emitter width biased at the minimum noise current density. All measurements were performed at $1.6 \mathrm{GHz}$. The series and parallel parasitics associated with probing pads were not de-embedded. Their contribution becomes comparable to the series base and emitter resistances of the transistor as the emitter length increases.

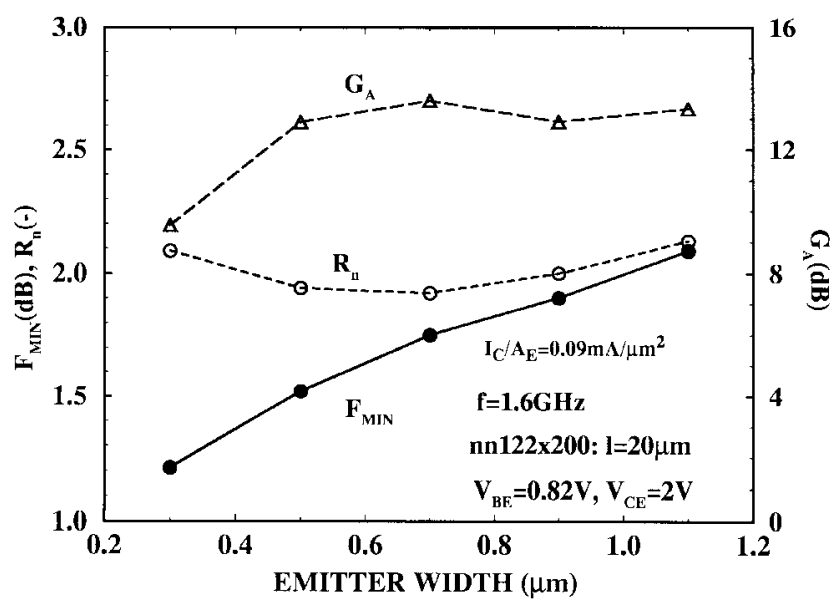

Fig. 4. ATN-NP5 on-wafer measured minimum noise figure $F_{\mathrm{MIN}}$, normalized (to $50 \Omega$ ) noise resistance $R_{n}$, and associated gain $G_{A}$, as a functions of emitter width for NT25 Si BJT's with single-stripe 20- $\mu \mathrm{m}$ long emitters, two base, and two collector contacts, biased at the minimum noise current density. The series and parallel parasitics associated with probing pads were not de-embedded. The label nn122x200 represents the standard NT25 npn nomenclature, where the first digit represents the number of emitter stripes (one), the second digit describes the number of base metal contacts (two) and the third digit stands for the number of collector contacts (two). $x$ describes the variable emitter width, whereas 200 stands for the emitter stripe length in tenths of micrometers (i.e., $20 \mu \mathrm{m}$ in this case).

to simultaneously compute $F_{\mathrm{MIN}}, f_{T}, f_{\mathrm{MAX}}, R_{n}$, and $Y_{\mathrm{sop}}$ as functions of $I_{C}$ in a single simulation run. The role of the simulator is to provide accurate, nonapproximated $Y$ parameters and $r_{B}\left(I_{C}\right)$ characteristics. The HSPICE-calculated noise parameters were found to agree within $0.25 \mathrm{~dB}$, up to $10 \mathrm{GHz}$, with those generated by LIBRA.

Measured and SGP-modeled $F_{\mathrm{MIN}}$, associated power gain $G_{A}, f_{T}$, and $f_{\mathrm{MAX}}$ are compared in Fig. 5 as functions of the collector current for a $0.65 \times 25 \mu \mathrm{m}^{2}$ single-poly bipolar transistor. The agreement is well within the typical on-wafer noise measurement error. The minimum noise current density is almost independent of $V_{\mathrm{CE}}$ and emitter length but increases 


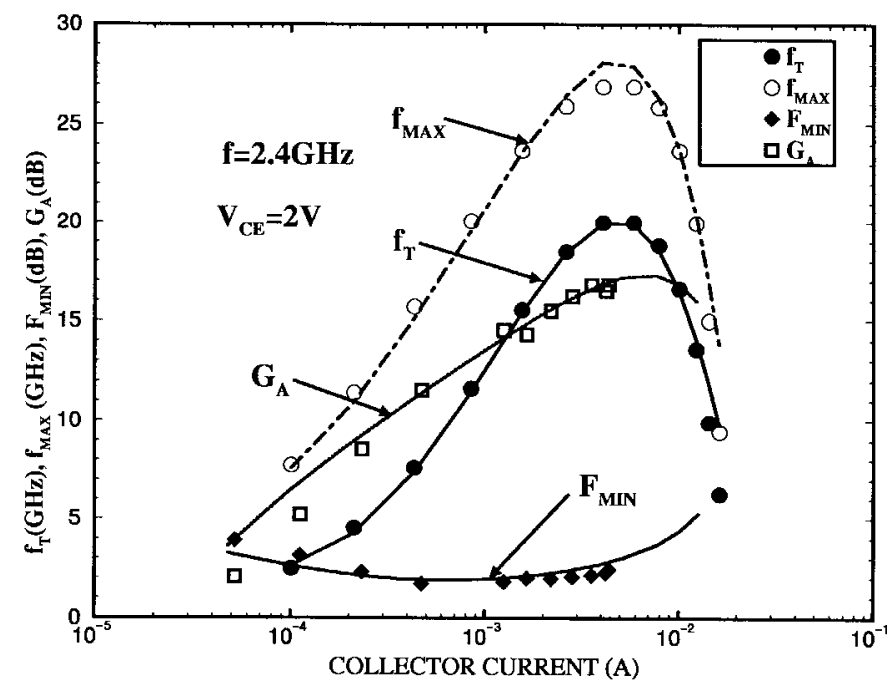

Fig. 5. Measured (symbols) and SGP-modeled (lines) $F_{\mathrm{MIN}}, f_{T}, f_{\mathrm{MAX}}$, $G_{A}$ versus $I_{C}$ characteristics for a $0.65 \times 25 \mu \mathrm{m}^{2}$ single-poly BiCMOS npn.

weakly with frequency. It is typically six to ten times smaller than the peak $f_{T}$ current density. The simulation results were obtained with a set of SGP model parameters extracted from dc and $S$ parameter measurements on the modeled device. No fitting of the noise measurement data was performed, thus confirming the accuracy of (7)-(9).

The measured and modeled frequency dependence of the noise parameters is plotted in Fig. 6 for a $0.5 \times 15 \mu \mathrm{m}^{2}$ SiGe HBT biased close to the minimum noise current density $J_{C}$. The rate of $F_{\mathrm{MIN}}$ degradation with frequency is inversely proportional to $f_{T}$, highlighting the requirement for transistors with large cutoff frequencies if low noise operation at high frequency is desired. The scatter in the measured data is due to the fact that the current NP5 system is unable to provide a controlled number of high impedance source states in a specified region of the Smith chart, as required by a small device with typical optimum source reflection coefficients larger than 0.8. In general, noise parameter measurements are not sufficiently accurate to provide a reliable extraction of the base resistance. As described in the next section, this can be more readily achieved from $Z$ parameter measurements.

\section{EXTRACTION OF TRANSISTOR NOISE PARAMETERS FROM $Y$ PARAMETER MEASUREMENTS}

Since noise-specific parameters are not present in (7)-(9), it follows that noise measurements are not necessary to obtain the transistor noise parameters. This remains valid even if correlation between the base and collector noise currents is considered [6]. Consequently, we propose to use (7)-(9) to determine the noise parameters of the transistor $F_{\mathrm{MIN}}$, $R_{n}, Y_{\text {sop }}$, from measured $Y$ parameters only, eliminating the need for lengthy and "noisy" on-wafer noise measurements. Such a possibility of measuring the $50-\Omega$ noise figure has been suggested earlier [11]. Here, it is extended to the complete set of noise parameters and it is verified in experiments. $r_{E}$ and $r_{B}\left(I_{C}\right)$, which are needed in addition to the $Y$ parameters, are obtained as follows. $r_{E}$ is determined from the frequency and collector current dependencies of the real part of the measured

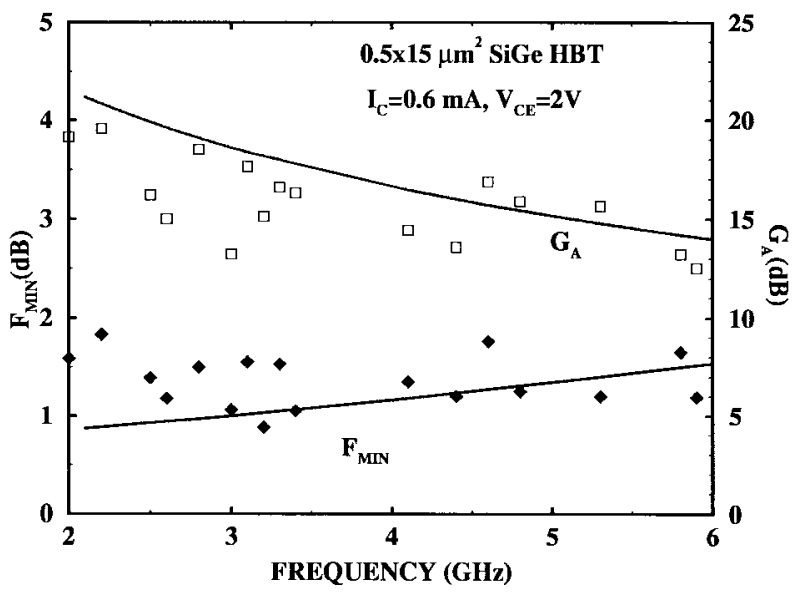

Fig. 6. Measured (symbols) and SGP-modeled (lines) $F_{\mathrm{MIN}}, G_{A}$ versus frequency characteristics for a $0.5 \times 15 \mu \mathrm{m}^{2}$ double-poly SiGe HBT at minimum noise bias.

$Z_{12}\left(f, I_{C}\right)$ characteristics, at low $V_{\mathrm{CE}}$ bias $(<1 \mathrm{~V})$ in order to avoid self-heating effects. First the real part of $Z_{12}\left(I_{C}\right)$ is averaged over the $0.1 \mathrm{GHz} \ldots 1 \mathrm{GHz}$ range for each bias point $I_{C}$. Then $r_{E}$ is extracted by linear interpolation from the $y$-axis intercept of the averaged $\operatorname{Re}\left\{Z_{12}\right\}$ versus $1 / I_{C}$ curve [12]. Similarly, the collector current-dependent base resistance $r_{B}\left(I_{C}\right)$ is obtained from the real part of $\left(Z_{11}-Z_{12}\right)$, averaged at frequencies below $1 \mathrm{GHz}$, where the impact of the substrate resistance is negligible. No modelrelated assumptions are made during the extraction about the shape of the $r_{B}\left(I_{C}\right)$ characteristics. An ac-measured, modelindependent, and noise-relevant $r_{B}\left(I_{C}\right)$ is thus plugged in (7)-(9) to obtain the measured noise parameters.

This technique was implemented in HP-EESOF's device characterization software package, ICCAP, and provides $f_{T}$, $f_{\mathrm{MAX}}, F_{\mathrm{MIN}}, R_{n}, Y_{\mathrm{sop}}$, and $G_{A}$ data from the same routine set of measured $Y$ parameters. It also elegantly solves the nontrivial noise parameter de-embedding problem since the $Y$ parameters have already been de-embedded using a conventional two-step shunt-series technique. Fig. 7 presents measured (from $Y$ parameters) and modeled data for the SiGe HBT emphasizing the impact of the $r_{B}\left(I_{C}\right)$ model on $F_{\mathrm{MIN}}$. The agreement between the $Y$ parameter-derived noise measurements and the conventional noise measurements in Fig. 6 is very good and there is no measurement scatter. Two base resistance models were considered in the simulated characteristics: a) the usual SGP base resistance model for the internal, bias-dependent base resistance term $r_{\mathrm{bi}}\left(I_{C}\right)$ (with $\mathrm{RBM}, \mathrm{RB}$, and IRB) to which a constant external term RBX is added (Fig. 8), and b) a constant resistance model with RB = RBM. In the case of the constant $r_{B}$ model, the base resistance was set to more accurately reflect the value at large current bias (peak $f_{T}$ ). In the latter case, the predicted noise figure is optimistic for most of the bias current range. In the case of the bias-dependent SGP base-resistance model, the agreement with the measurements is excellent. The plots indicate that the impact of the base resistance model on the minimum noise figure is only significant at collector currents below the minimum noise current density, where the transistor noise is 


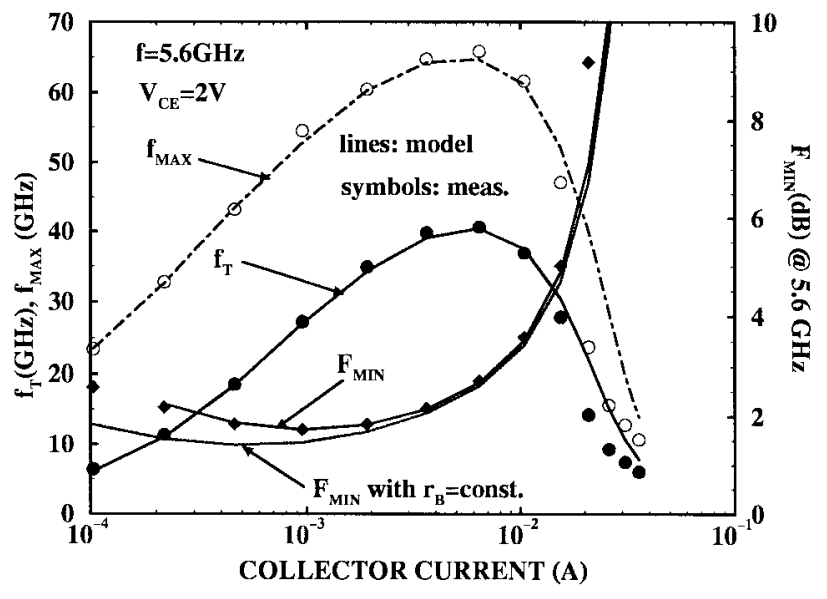

Fig. 7. Measured (from $Y$ parameters) versus SGP-modeled $f_{T}, f_{\mathrm{MAX}}$, and $F_{\mathrm{MIN}}$ versus $I_{C}$ characteristics for $0.5 \times 15 \mu \mathrm{m}^{2} \mathrm{SiGe} \mathrm{HBT}$, showing impact of the base resistance model on the minimum noise figure.

primarily of thermal origins. Even in that range, the difference is a few tenths of one $\mathrm{dB}$, i.e., within the typical accuracy of on-wafer noise measurements. Based on these experiments and simulations, one expects that, at least for narrow emitter transistors, as is the case of state-of-the-art Si bipolar devices, extracting the base resistance from noise figure measurements alone is of questionable accuracy. An alternative approach is to combine noise and $S$ parameter measurements in the extraction of the small signal parameters [6]. The scatter in the noise measurement can still lead to nonphysical small signal parameters, particularly so if optimization is employed.

In this paper, the SGP base resistance model is obtained as follows: RB is analytically calculated from dc pinchedbase resistance measurements and transistor geometry, RBM is set to $0.001 \Omega$, while IRB and RBX are extracted from the $\operatorname{Re}\left\{Z_{11}-Z_{12}\right\}$ and $f_{\mathrm{MAX}}\left(I_{C}\right)$ characteristics, as described above. This combined dc and ac-extraction technique, involving highly accurate measurements, appears to provide $r_{B}\left(I_{C}\right)$ characteristics that, coupled with the appropriate distributed equivalent circuit (Fig. 8), match both the ac (i.e., $\operatorname{Re}\left\{Z_{11}-\right.$ $\left.\left.Z_{12}\right\}, f_{\mathrm{MAX}}\right)$ and the noise behavior of the transistor. The very same RB and RBX values are used in the HICUM model, but the $r_{\mathrm{bi}}\left(I_{C}\right)$ dependence follows device physics more closely [2].

\section{Scalable Model ImPlementation AND VerificATION}

The ability to predict the impact of (statistical) emitter width and length variations on the noise parameters depends on the availability of a physically based, scalable compact model. In this paper, geometry- and process-scalable variants of HICUM [1] and of the SGP models have been used. The scalable HICUM model has been recently described in detail [1]. In the case of the SGP model, the subcircuit presented in Fig. 8 was employed. In addition to the core vertical npn model, it uses external diodes, resistors, and capacitors to reflect the distributed nature of the base resistance and the physical partitioning of the base-emitter and base-collector capacitances [13]. The main features of the scalable SGP model are summarized below.

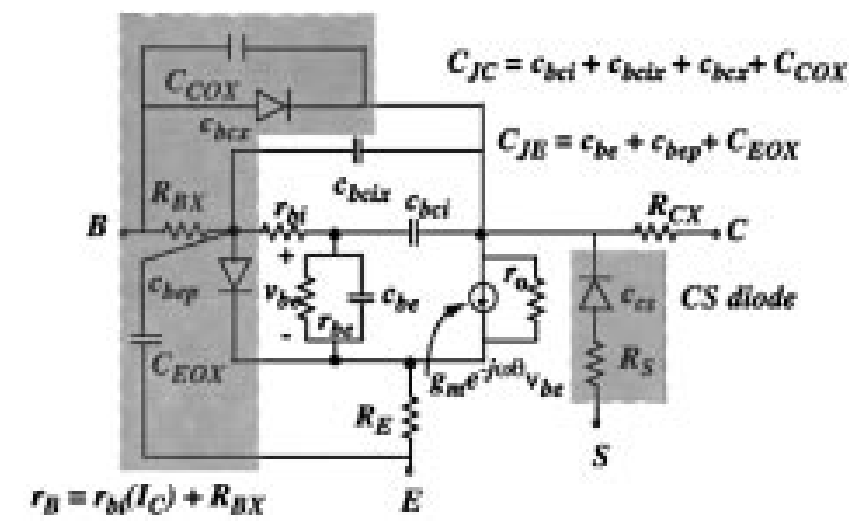

Fig. 8. Small signal equivalent circuit used in the scalable SGP model. Circuit elements added to the core SGP model have been highlighted.

i) An external base-emitter diode is used to account for the different grading coefficients of the voltage dependence of the area and periphery components of the baseemitter capacitance.

ii) The part of the base-collector capacitance corresponding to the selectively implanted collector (SIC) region $c_{\mathrm{bci}}+c_{\mathrm{bcix}}$ is captured in the internal transistor equivalent circuit whereas the epitaxial part of the collector capacitance $c_{\mathrm{bcx}}$ is modeled using the external basecollector diode.

iii) The base resistance is split in a bias-dependent $r_{\mathrm{bi}}$ and a bias-independent term $R_{\mathrm{BX}}$.

iv) In order to account for the series resistance $R_{S}$ associated with the collector-substrate region, and to allow for the physical partitioning of the collector resistance and collector-substrate capacitance, an external collectorsubstrate diode is employed. Even though the value of $R_{S}$ varies with the number and position of the substrate contacts, its accurate extraction is important as it affects $f_{\mathrm{MAX}}$ and, depending on the extraction technique, $r_{B}$ and even $f_{T}$.

v) Bias-independent oxide capacitances associated with the emitter poly overlap of the base $C_{\mathrm{EOX}}$ and with the base poly overlap of the field oxide $C_{\mathrm{COx}}$ are deployed between the base and the emitter and between the base and the collector, respectively.

vi) The scaling of the low current transit time parameter TF with emitter size is implemented as in HICUM using (8) of [1].

vii) The large current transit time parameter ITF is scaled with emitter area and also accounts for collector current spreading via the same function $f_{\mathrm{Cs}}$ as HICUM, described by (20) of [1].

viii) Weak avalanche multiplication is implemented using the same equation as in HICUM.

ix) Process scaling is achieved by physically relating area and periphery components of junction capacitances, saturation currents, transit time parameters, internal and external resistances to the pinched base resistance, SIC region resistance, poly sheet resistances, and oxide capacitances. 


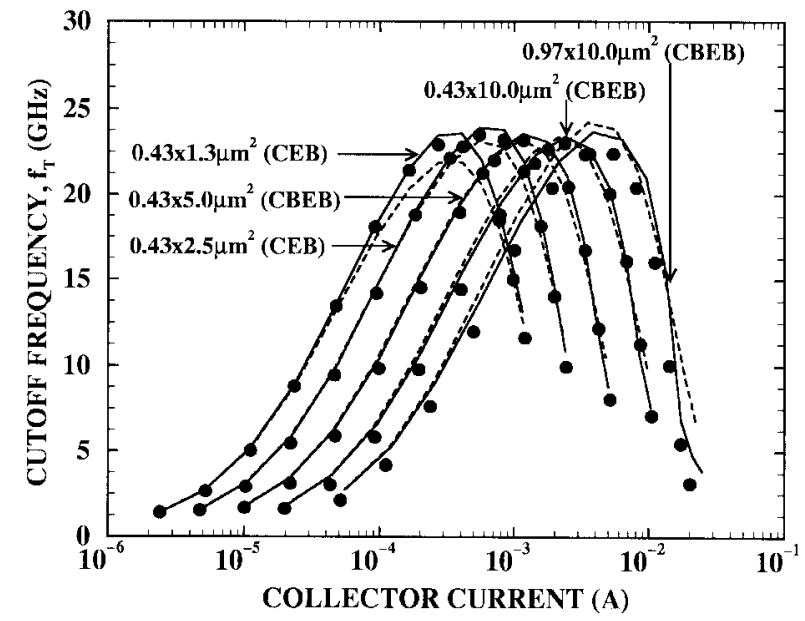

Fig. 9. Measured (symbols) versus HICUM- (solid lines) and SGP- (dashed lines) modeled $f_{T}$ versus $I_{C}$ characteristics for "nominal" NT25 Si BJT's biased at $V_{\mathrm{CE}}=1 \mathrm{~V}$.

x) All model parameters are derived as functions of emitter geometry and number of emitter, base, and collector contacts, and these functions are implemented as a preprocessor in HSPICE and ELDO decks.

Measured and HICUM- and SGP-modeled $f_{T}$ and $F_{\mathrm{MIN}}$ characteristics are investigated in Figs. 9 and 10, respectively, for different transistor geometries on a "nominal" NT25 wafer at $V_{\mathrm{CE}}=1 \mathrm{~V}$. The error between measured and SGP and HICUM predicted $f_{T}$ and $F_{\mathrm{MIN}}$ characteristics is less than $7 \%$ over all geometries and over the entire bias current range up to more than two times the peak $f_{T}$ current density. Included in this error are random emitter width variations across the die, typically up to $5 \%$, as well as measurement and de-embedding errors, estimated at 2-3\%. This kind of accuracy is also obtained for other $V_{\mathrm{CE}}$ bias values ranging from $0.5 \mathrm{~V}$ to $3.3 \mathrm{~V}$. The SGP and HICUM model parameters were calculated from the same set of process and geometry data. There was no individual "tweaking" of the model parameters to better fit the characteristics of each transistor. In the low-noise regime, HICUM- and SGP-modeled transistor characteristics are practically indistinguishable. Because of the more sophisticated transit time model, HICUM is slightly more accurate than the SGP model with respect to $f_{T}$ scaling with transistor geometry at moderate and large currents. In the high-current regime, the dc characteristics are affected by self-heating effects, but the latter do not significantly impact $f_{T}$ versus $I_{C}$ characteristics. At present, self-heating effects are only accounted for in the HICUM model. The results in Figs. 9 and 10 establish the accuracy of the scalable HICUM and SGP models and demonstrate their applicability in a new bipolar circuit design philosophy where the transistor geometry becomes part of the design space.

\section{Application to LNA Design}

Finally, the noise parameter equations and the scalable SGP model are applied to the design of tuned LNA's at 1.9, 2.4, and $5.8 \mathrm{GHz}$ using $\mathrm{Si}$ and $\mathrm{SiGe}$ double-poly bipolar transistors. Present-day tuned LNA and mixer circuits for wireless systems

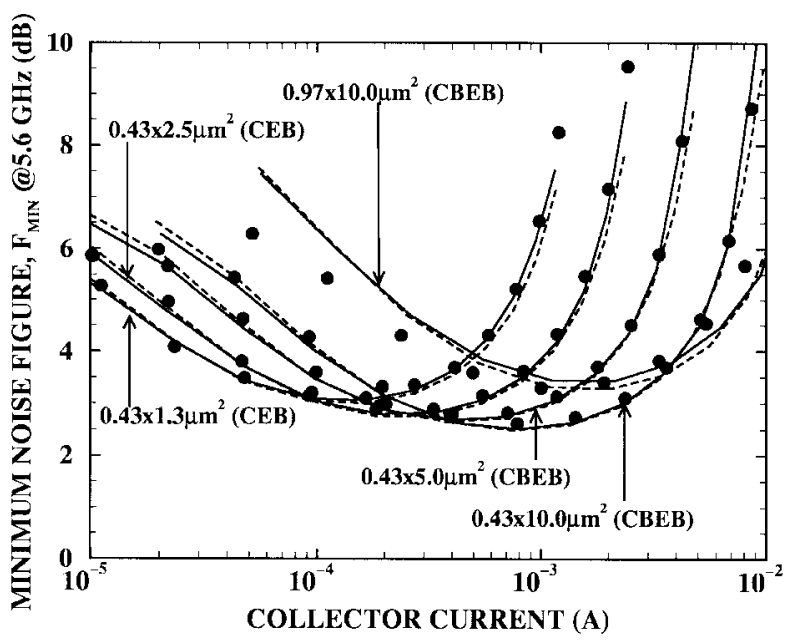

Fig. 10. Measured (symbols) versus HICUM- (solid lines) and SGP- (dashed lines) modeled $F_{\mathrm{MIN}}$ versus $I_{C}$ characteristics for "nominal" NT25 Si BJT's biased at $V_{\mathrm{CE}}=1 \mathrm{~V}$.

often trade off noise and input impedance matching [14], [15]. This tradeoff is sometimes caused by the fact that the size of bipolar transistors is not traditionally considered as a design variable which can be continuously varied and optimized in a SPICE deck just like any other circuit element. In other situations, typical for GaAs-based technologies where the importance of optimal noise biasing and matching is well established [16]-[18], the lack of sufficiently accurate physical and scalable transistor models make such an approach unreliable. In the case of Si MOSFET's, where transistor width is a ubiquitous design variable, high-frequency noise performance is at best poorly understood and modeled. To the best of these authors' knowledge, an algorithmic design methodology with an optimal and unique solution to the noise- and inputimpedance matching problem has not yet been developed. Instead, a passive network is designed around a given transistor in order to achieve noise matching and/or impedance matching [14], [15]. The passive network itself contributes losses and hence degrades the noise figure. The losses in the passive network increase as the network becomes more complicated and are particularly severe on silicon substrates. For this very reason, in Si RF IC's the matching circuitry is typically left off-chip [15].

Examination of (11) shows that the optimum noise conductance $G_{\text {SOP }}$ is different from the input conductance, whereas the optimum noise susceptance $B_{\mathrm{SOP}}$ is equal to the complex conjugate of the input susceptance of the device. This is a fundamental characteristic of both bipolar and field-effect transistors and underlies the requirement for separate treatment of matching the real part of the input impedance and the real part of the optimum noise impedance. The goal of the new design philosophy detailed below is to obtain simultaneous noise and input impedance matched circuits. It is applicable to both bipolar and FET circuits.

In order to minimize the losses in the passive network around the transistor, the size of the transistor is first designed so that the transistor becomes noise matched to the characteristic impedance of the system, typically $50 \Omega$, at the 


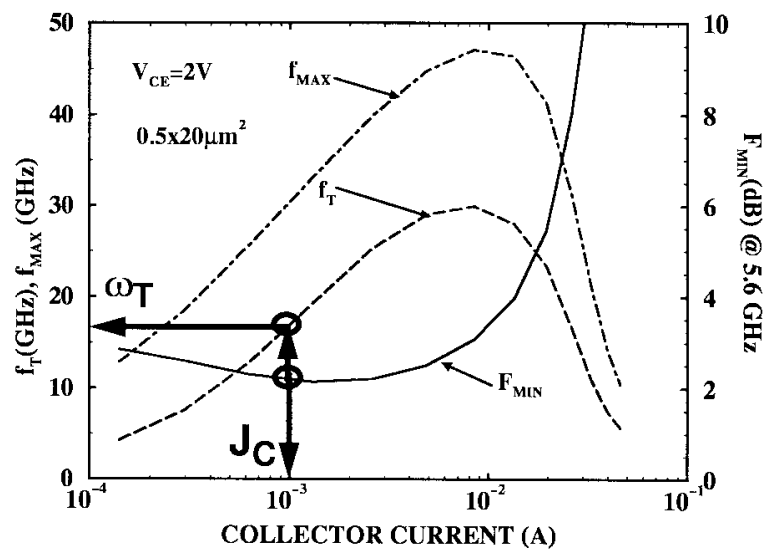

Fig. 11. SGP-modeled $f_{T}, f_{\mathrm{MAX}}$, and $F_{\mathrm{MIN}}$ as functions of the collector current for the $0.5-\mu \mathrm{m}$ emitter, NT25 double-poly Si bipolar process, illustrating the technique for identifying the minimum noise current density $J_{C}$ and the corresponding cutoff frequency $\omega_{T}$ used in circuit design.

desired frequency. Because the transistor is an active device, noise matching is achieved without losses and without noise figure degradation. Since the task of noise matching is removed from the passive network, the latter becomes simpler and less lossy. This can be labeled the low-noise-matched transistor design stage. Finally, to complete the circuit, a minimal passive network with two low-loss inductors is designed to provide input impedance matching with the lowest possible degradation of the overall noise figure. This corresponds to the passive matching network design stage.

\section{A. Low-Noise-Matched Transistor Design}

The transistor design stage first involves finding the optimal noise current density $J_{C}$ from (9) using the HSPICE deck, as shown in Fig. 11. As mentioned in Section II, the minimum noise figure and the optimum noise current density are practically independent of emitter length. The emitter length is then adjusted so that the optimum source resistance $R_{\text {sop }}$ equals $Z_{0}(50 \Omega)$ at the minimum noise current density and at frequency $f$, as expressed in (12) and illustrated schematically in Fig. 12. After these two steps, the transistor size and its bias current are determined. The real part of the optimum noise impedance is now matched to $50 \Omega$ without having degraded the minimum noise figure.

\section{B. Passive Matching Network Design}

An emitter inductor $L_{E}$ is added to match the real part of the input impedance to $Z_{0}$ [14], [18]

$$
L_{E} \cong \frac{Z_{0}}{2 \pi f_{T}} \text {. }
$$

It can be demonstrated using the theory of correlated noise sources in series-series feedback circuits [16] that, if lossless, $L_{E}$ does not change the value of $R_{\mathrm{SOP}}$ but that it does affect the optimum source reactance $X_{\mathrm{SOP}}$. For the combined transistor-emitter inductor structure, the latter becomes

$$
X_{\text {sop }} \cong \frac{\frac{n R_{n} f_{T}}{2 f}}{\frac{I_{C}}{2 V_{T}}\left(r_{E}+r_{B}\right)\left(1+\frac{f_{T}^{2}}{\beta_{0} f^{2}}\right)+\frac{n^{2}}{4}\left(1+\frac{f_{T}^{2}}{\beta_{0} f^{2}}\right)}
$$

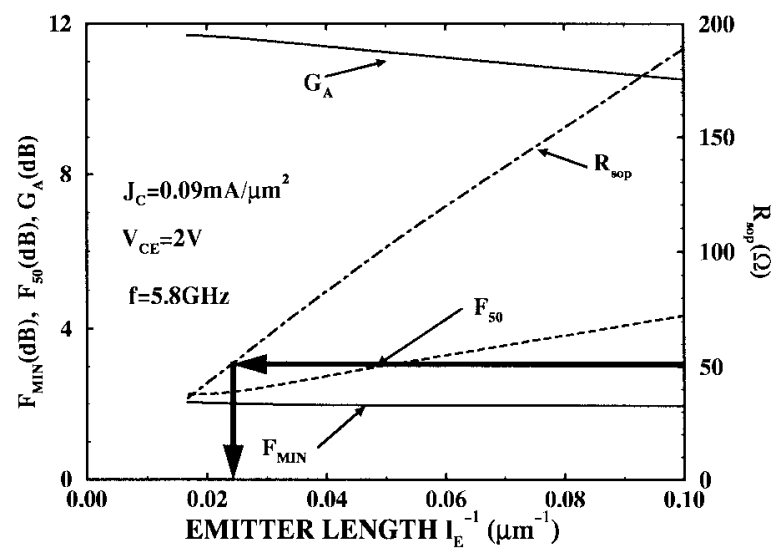

Fig. 12. SGP-modeled noise parameters as functions of the emitter length $l_{E}$ for the $0.5-\mu \mathrm{m}$ emitter, NT25 double-poly Si bipolar process. $F_{50}$ is the noise figure in a 50- $\Omega$ system. Selection of the emitter length corresponding to an optimum noise source resistance of $50 \Omega$ is also sketched.

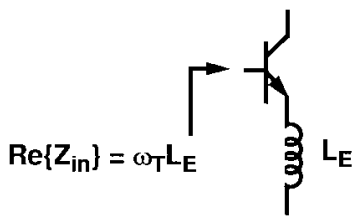

(a)

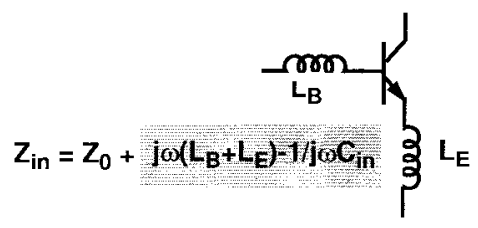

(b)

Fig. 13. Illustration of the input impedance matching procedure: (a) adding an emitter inductor to transform the real part of the input impedance to $50 \Omega$ and (b) adding an inductor in the base to simultaneously cancel out the imaginary part of the input impedance and of the optimum noise impedance.

$$
-2 \pi f L_{E}
$$

Simultaneous noise and input impedance match is finally obtained by connecting an inductor $L_{B}$ in the base. It cancels out the reactance due to the input capacitance $C_{\text {in }}$ of the device, and, at the same time, it transforms the optimum noise reactance of the amplifier to $0 \Omega$

$$
L_{B} \cong \frac{1}{\omega^{2} C_{\text {in }}}-L_{E} \text {. }
$$

The matching network design stage is schematically shown in Fig. 13.

This design methodology guarantees optimal noise and input impedance match with the simplest matching network. An LNA design can be completed by adding a suitable matching network in the collector in order to maximize the power gain [18], [19]. Simulation results obtained with LIBRA are shown in Fig. 14. Table I illustrates several design examples at 1.9, 2.4, and 5.8 GHz. Ideal (lossless) inductors were assumed. As expected from the noise parameter equations, the optimal transistor size and bias current decrease with increasing frequency. At $5.8 \mathrm{GHz}$ the SiGe HBT version is 
TABLE I

0.5- $\mu \mathrm{m}$ Multiple-Finger Emitter, NT25 ANd SiGe HBT SingLE-Transistor LNA Design Data at Minimum Noise Bias And $V_{\mathrm{CE}}=2 \mathrm{~V}$. Ideal Inductors Were UsED with No OUtPut Matching. CutofF and Oscillation Frequency Values Are at the Low-Noise Bias Current

\begin{tabular}{l|l|l|l|l}
\hline & \multicolumn{1}{|c|}{$\begin{array}{c}\text { Si npn } \\
1.9 \mathrm{GHz}\end{array}$} & \multicolumn{1}{c|}{$\begin{array}{c}\text { Si npn } \\
2.4 \mathrm{GHz}\end{array}$} & \multicolumn{1}{c|}{$\begin{array}{c}\text { Si npn } \\
5.8 \mathrm{GHz}\end{array}$} & \multicolumn{1}{c}{$\begin{array}{c}\text { SiGe HBT } \\
5.8 \mathrm{GHz}\end{array}$} \\
\hline \hline $\mathrm{w}_{\mathrm{E}}, 1_{\mathrm{E}}$ & $4 \times 0.5 \times 28 \mu \mathrm{m}^{2}$ & $4 \times 0.5 \times 23 \mu \mathrm{m}^{2}$ & $2 \times 0.5 \times 20 \mu \mathrm{m}^{2}$ & $3 \times 0.5 \times 15 \mu \mathrm{m}^{2}$ \\
\hline $\mathrm{I}_{\mathrm{C}}$ & $3.1 \mathrm{~mA}$ & $2.5 \mathrm{~mA}$ & $1.7 \mathrm{~mA}$ & $1.4 \mathrm{~mA}$ \\
\hline $\mathrm{f}_{\mathrm{T}}$ & $11 \mathrm{GHz}$ & $12 \mathrm{GHz}$ & $15 \mathrm{GHz}$ & $20 \mathrm{GHz}$ \\
\hline $\mathrm{f}_{\mathrm{MAX}}$ & $31 \mathrm{GHz}$ & $33 \mathrm{GHz}$ & $38 \mathrm{GHz}$ & $45 \mathrm{GHz}$ \\
\hline $\mathrm{L}_{\mathrm{E}}$ & $1.04 \mathrm{nH}$ & $1.01 \mathrm{nH}$ & $0.54 \mathrm{nH}$ & $0.45 \mathrm{nH}$ \\
\hline $\mathrm{L}_{\mathrm{B}}$ & $2.30 \mathrm{nH}$ & $1.84 \mathrm{nH}$ & $0.60 \mathrm{nH}$ & $1.09 \mathrm{nH}$ \\
\hline $\mathrm{F}_{\min }$ & $0.87 \mathrm{~dB}$ & $0.98 \mathrm{~dB}$ & $2.02 \mathrm{~dB}$ & $1.43 \mathrm{~dB}$ \\
\hline $\mathrm{F}_{50}$ & $0.88 \mathrm{~dB}$ & $0.98 \mathrm{~dB}$ & $2.02 \mathrm{~dB}$ & $1.45 \mathrm{~dB}$ \\
\hline $\mathrm{G}_{\mathrm{A}}$ & $15.2 \mathrm{~dB}$ & $14.2 \mathrm{~dB}$ & $11.1 \mathrm{~dB}$ & $14.0 \mathrm{~dB}$ \\
\hline $\mathrm{IS}_{11} \mathrm{I}$ & $-38 \mathrm{~dB}$ & $-31 \mathrm{~dB}$ & $-42 \mathrm{~dB}$ & $-33 \mathrm{~dB}$ \\
\hline
\end{tabular}

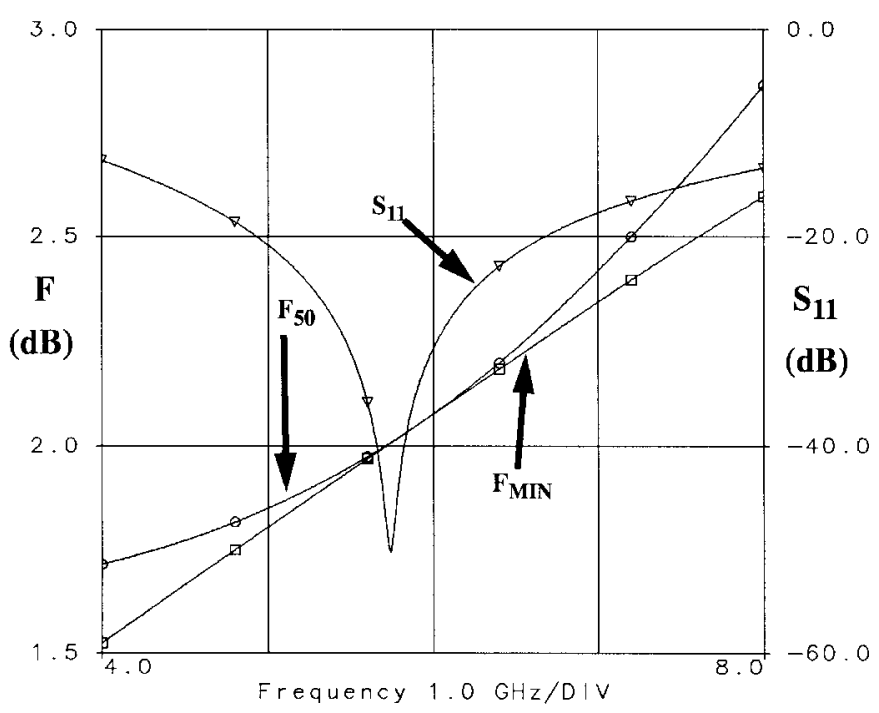

Fig. 14. LIBRA simulated $F_{\mathrm{MIN}}, F_{50}$, and $\left|S_{11}\right|$ for a 5.8-GHz LNA employing a $2 \times 0.5 \times 20 \mu \mathrm{m}^{2}$ Si double-poly transistor at $V_{\mathrm{CE}}=2 \mathrm{~V}$.

predicted to have lower noise (mostly due to the higher $f_{T}$ ) and higher gain (because of higher $f_{\mathrm{MAX}}$ ) while dissipating less power than the corresponding Si bipolar circuit.

Single transistor test structures with emitter inductors only, and with both base and emitter inductors, were fabricated in the NT25 Si bipolar process at 1.9, 2.4, and $5.8 \mathrm{GHz}$. The measured data confirmed the simultaneous noise and input impedance match. The input return loss was better than $19 \mathrm{~dB}$ in all cases. Fig. 15 shows the measured input impedance and associated gain for a single transistor structure with on-chip emitter and base inductors as a function of the pinchedbase resistance (PBR). The latter was intentionally varied between the process extremes to examine its impact on the manufacturability and yield of tuned LNA's. The measured noise figure varied by $0.2 \mathrm{~dB}$ over the estimated process spread of the PBR. Using (13), the small variation in noise figure can be explained by the opposite effects that the PBR has

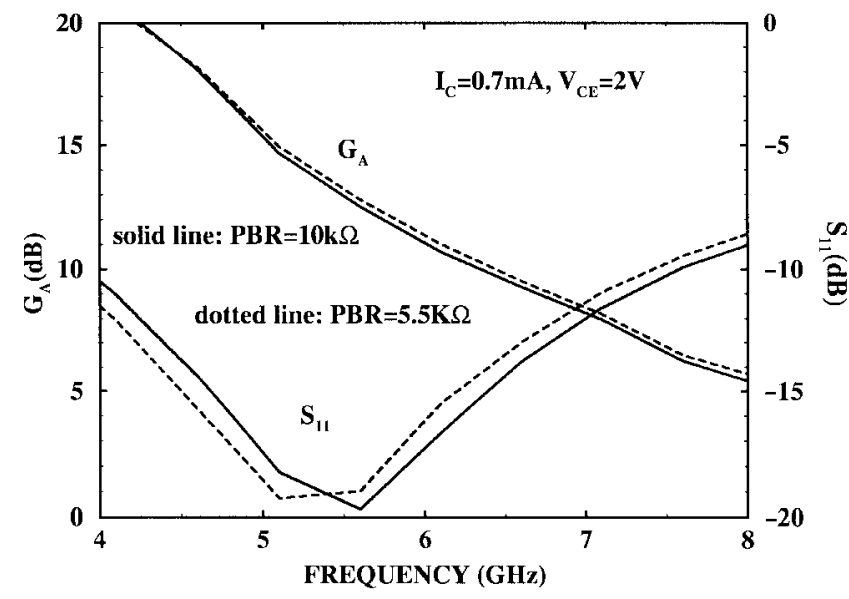

Fig. 15. Measured input impedance and associated gain for a single transistor structure with on-chip emitter and base inductors as a function of pinched-base resistance (PBR) for NT25 bipolar transistors.

on $f_{T}$ and $r_{B}$ which tend to cancel out in the same manner as for the maximum oscillation frequency. When compared to the ideal inductor simulation data, the finite $Q$ of the fabricated on-wafer inductors, typically seven to ten, degraded the noise figure by $0.7-1.4 \mathrm{~dB}$. It was found that the base inductor contributed $0.4-0.7 \mathrm{~dB}$ to the measured overall noise figure, depending on the inductor value and transistor size. This degradation is proportionally more severe at the lower frequencies $(2.4$ and $1.9 \mathrm{GHz})$ where large transistor sizes and large inductor values are required, as indicated in Table I.

The impact of the finite $Q$ of the inductor can be reasonably well accounted for in simulations if an appropriate inductor model is employed [20]. Also, in the previous analytical expressions, the series resistance of the base and emitter inductors can be respectively incorporated into the base and emitter resistances of the transistor. The flow and the validity of the low-noise design methodology remain unaltered. As an example, for a 5.8-GHz tuned cascode LNA fabricated in NT25 and presented in detail elsewhere [19], it was found that the difference between the measured $(4.2 \mathrm{~dB})$ and simulated ( $3.8 \mathrm{~dB}$ ) noise figures was about $10 \%$. In this case, the common base transistor contributed an extra $1 \mathrm{~dB}$ to the overall noise figure of the amplifier. When the base inductor was left off chip, the measured noise figure decreased to $3.8 \mathrm{~dB}$. Clearly, improvement in inductor $Q$ 's by using thicker $(>3 \mu \mathrm{m})$ goldbased metallization and thicker dielectric isolation between the inductor and the Si substrate can have as significant an impact on Si low-noise amplifier performance as moving to the next generation of SiGe-based technology. With similar inductor technology, SiGe still provides $0.5-0.6 \mathrm{~dB}$ lower noise figure at $5.8 \mathrm{GHz}$.

\section{CONCLUSION}

The capability of scalable variants of the SGP and HICUM models to accurately predict noise parameters was demonstrated using measurements and simulations on three different high-speed $\mathrm{Si}$ and SiGe technologies. A technique for extracting noise parameters from $S / Y$ parameter measurements was described. Finally, a design methodology for low-noise amplifiers was illustrated with the goal of optimizing the 
emitter geometry in order to minimize matching circuit losses and overall noise figure. In essence, this design methodology allows for the design of transistors that have the real part of the optimum noise impedance equal to $50 \Omega$ at the desired frequency of operation.

\section{ACKNOWLEDGMENT}

The authors would like to thank T. MacElwee for the BiCMOS wafers and the staff of Nortel Semiconductors for providing the NT25 double-poly Si npn wafers.

\section{REFERENCES}

[1] M. Schroter and D. J. Walkey, "Physical modeling of lateral scaling in bipolar transistors," IEEE J. Solid-State Circuits, vol. 31, pp. 1484-1492, Oct. 1996.

[2] H. Stubing, H.-M. Rein, and M. Schroter, "A compact physical largesignal model for high-speed bipolar transistors at high current densities part I and part II," IEEE Trans. Electron Devices, vol. 34, pp. 1741-1761, Aug. 1987.

[3] H. Hillbrand and P. H. Russer, "An efficient method for computer aided noise analysis of linear networks," IEEE Trans. Circuits Syst., vol. CAS-23, pp. 235-238, Apr. 1976.

[4] G. D. Vendelin, A. M. Pavio, and U. L. Rhode, Microwave Circuit Design Using Linear and Nonlinear Techniques. New York: Wiley, ch. 2, pp. 93-97, 1990.

[5] G. Dambrine, H. Happy, F. Danneville, and A. Cappy, "A new method for on wafer noise measurement," IEEE Trans. Microwave Theory Technol., vol. 41, pp. 375-381, Mar. 1993.

[6] J. P. Roux, L. Escotte, R. Plana, J. Graffeuil, S. L. Delage, and H. Blanck, "Small-signal and noise model extraction technique for heterojunction bipolar transistor at microwave frequencies," IEEE Trans. Microwave Theory Technol., vol. 43, pp. 293-297, Feb. 1995.

[7] R.-H. Yang, T. M. Liu, T.-Y. Chiu, and V. D. Archer, "Direct measurement of base drift field in bipolar transistors," IEEE Electron Device Lett., vol. 13, pp. 276-278, May 1992.

[8] D. L. Harame, L. Larson, M. Case, S. Kovacic, S. Voinigescu, T Tewksbury, D. Nguyen-Ngoc, K. Stein, J. Cressler, S.-J. Jeng, J. Malinowski, R. Groves, E. Eld, D. Sunderland, D. Rensch, M. Gilbert, K. Schonenberg, D. Ahlgren, S. Rosenbaum, J. Glenn, and B. Meyerson, "SiGe HBT technology: Device and application issues," in IEDM Tech. Dig., Dec. 1995, pp. 731-734.

[9] S. P. Voinigescu, S. W. Tarasewicz, T. MacElwee, and J. Ilowski, “An assessment of the state-of-the-art $0.5 \mu \mathrm{m}$ bulk CMOS technology for RF applications," in IEDM Tech. Dig., Dec. 1995, pp. 721-724.

[10] S. P. Voinigescu, P. B. Rabkin, C. A. T. Salama, and P. Blakey, "2D numerical investigation of the impact of compositional grading on the performance of submicrometer Si/SiGe p-MOSFET's," IEEE Trans. Electron Devices, vol. ED-42, pp. 1039-1046, June 1995 and S. P. Voinigescu, "VLSI compatible Si/SiGe/Si p-MOSFET's," Ph.D. dissertation, Chap. $3\left(f_{T}, f_{\mathrm{MAX}}\right.$, and $F_{\mathrm{MIN}}$ scaling in $\mathrm{Si}$ and $\mathrm{SiGe}$ MOSFET's), University of Toronto, Aug. 1994.

[11] F. Herzel and D. Heinemann, "A novel approach to HF-noise characterization of heterojunction bipolar transistors," in Simulation of Semiconductor Devices and Processes, H. Ryssel and P. Pichler, Eds., vol. 6, pp. 98-101, 1995.

[12] S. J. Spiegel, D. Ritter, R. A. Hamm, A. Feygenson, and P. R. Smith, "Extraction of the InP/GaInAs heterojunction bipolar transistor smallsignal equivalent circuit," IEEE Trans. Electron Devices, vol. ED-42, pp. 1059-1064, June 1995.

[13] A. Koldehoff, M. Schroter, and H.-M. Rein, "A compact bipolar transistor model for very-high-frequency applications with special regard to narrow emitter stripes and high current densities," Solid-State Electron., vol. 36, pp. 1035-1048, July 1993.

[14] F. McGrath, K. Jackson, E. Heaney, A. Douglas, W. Fahey, R. G. Pratt, and T. Begnoche, "A 1.9-GHz GaAs chip set for the personal handyphone system," IEEE Trans. Microwave Theory Technol., vol. 43, pp. 1733-1744, July 1995.

[15] A. N. Karanicolas, "A 2.7-V 900-MHz CMOS LNA and mixer," IEEE J. Solid-State Circuits, vol. 31, pp. 1939-1944, Dec. 1996.

[16] S. Voinigescu and D. Neculoiu, "Noise in electronic receivers-Design problems," (in Romanian), Lecture notes, Polytechnic Institute of Bucharest, ch. 4: Design of Low-Noise Feedback Amplifiers, pp. 51-64, 1989.
[17] B. Hughes, "Designing FET's for broad noise circles," IEEE Trans. Microwave Theory Technol., vol. 41, pp. 190-198, Feb. 1993.

[18] K. W. Kobayashi, A. K. Oki, L. T. Tran, and D. C. Streit, "Ultra-low dc power GaAs HBT S-band low noise amplifiers," in IEEE Microwave and Millimeter-Wave Monolithic Circuits Symp. Dig., 1995, pp. 73-76.

[19] S. P. Voinigescu and M. C. Maliepaard, "5.8 GHz and $12.6 \mathrm{GHz} \mathrm{Si}$ bipolar MMIC's," in ISSCC-Dig., Feb. 1997, pp. 372-373.

[20] J. Long and M. Copeland, "Modeling, characterization and design of monolithic inductors for silicon RFIC's," in CICC Dig., May 1996, pp. $185-188$.

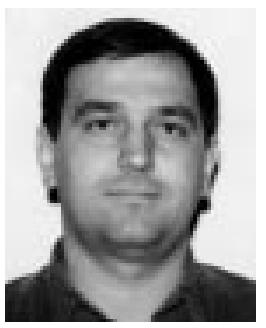

Sorin P. Voinigescu (M'95) graduated in 1984 with the M.Sc. degree in electronics from the Polytechnic Institute of Bucharest, Romania. He received the $\mathrm{Ph} . \mathrm{D}$. degree in electrical and computer engineering from the University of Toronto, Canada, in 1994. His Ph.D. dissertation was on the design and fabrication of VLSI compatible Si/SiGe p-MOSFET's.

Between 1984 and 1991 he worked in R\&D and in academia in Bucharest. He designed circuits for an $11-\mathrm{GHz}$ digital radio, co-developed a selfaligned process for GaAs Schottky and varactor diodes, and investigated the numerical modelling of quantum effects in AlGaAs/GaAs MODFET's and of heavy-doping effects in Si PIN diodes. $\mathrm{He}$ also lectured at the Polytechnic Institute of Bucharest on the design of microwave semiconductor devices and integrated circuits. In 1994, he joined NORTEL (Northern Telecom), in Ottawa, Canada, where he has been responsible for high-frequency characterization and compact model extraction for $\mathrm{Si}, \mathrm{SiGe}$, and $\mathrm{SiC}$ devices. His current duties are in the design of Si-based MMIC's for wireless and broad-band fiber optics transceivers at $5 \mathrm{GHz}$ and $10 \mathrm{~Gb} / \mathrm{s}$, respectively. He has authored or co-authored more than 25 refereed technical papers spanning the simulation, modelling, design, and fabrication of GaAs- and Si-based heterostructure devices and circuits.

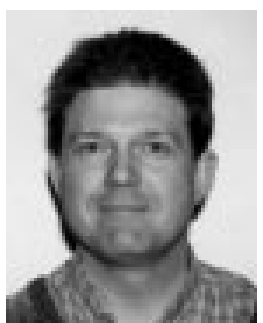

Michael C. Maliepaard (M'89) received the B.Sc. degree in physics from Queen's University, Kingston, Canada, in 1985 and the Ph.D. degree in physics from Cambridge University, U.K., in 1989.

He joined Nortel in 1989 to work as a reliability physicist in their Silicon Process Technology Department. His work focused on providing ESD protection, latch-up immunity and hotcarrier characterization for CMOS and BiCMOS processes. He also provided design, modeling, and characterization of EPROM and fuse PROM core cells. After a period working on parametric yield issues for the silicon fab, he joined the Technology Access and Applications Department where he is currently investigating the potential of CMOS technologies for implementing $\mathrm{RF}$ circuits. His research interests include RF circuit design, RF integration issues, and high-speed semiconductor technologies.

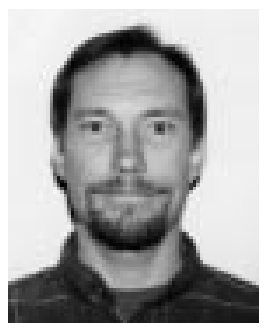

Jonathan L. Showell (S'90-M'95) received the B.Eng. and M.Eng. degrees in engineering physics from McMaster University, Hamilton, ON, Canada, in 1990 and 1994, respectively. As part of his graduate studies, he worked on the fabrication and characterization of $\mathrm{InP} / \mathrm{InGaAsP}$ devices for an optoelectronic integrated circuit (OEIC).

During this time, he made trips to the National Research Council of Canada, in Ottawa, Canada, as a Visiting Graduate Researcher, where he developed fabrication process steps for the solid-state optoelectronics consortium (SSOC). In 1994 he joined Northern Telecom, Ottawa, working on hot carrier injection reliability of CMOS devices in a BiCMOS line. He is now with the Technology Access and Applications Group at Nortel Technology, working primarily on the parameter extraction of high-speed bipolar and heterojunction transistors for accurate technology assessment. His interests include high-speed technologies, RF circuit design, and accurate RF electrical measurements. 


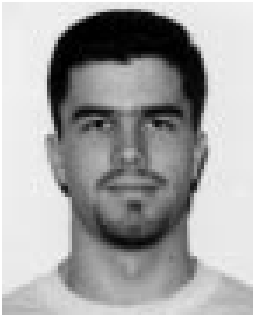

Greg E. Babcock is completing the undergraduate degree in electrical engineering at Queen's University, Kingston, ON, Canada.

In May 1996 he joined Nortel Technology, Ottawa, ON, for a 16-month internship, where he performed high-frequency $(0.1-26.1 \mathrm{GHz})$ measurements on high-speed bipolar and FET technologies. In addition, he has aided in the parameter extraction and verification of the aforementioned technologies. During his stay with Nortel Technology he has fostered a strong interest in device physics, which he intends to apply towards RF circuit design.

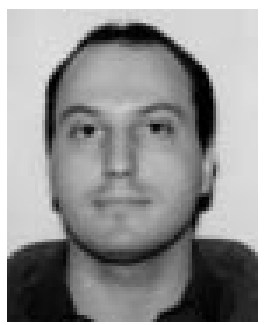

David Marchesan (M'96) received the B.A.Sc degree in physics from the University of Waterloo, ON, Canada, in 1993 and the M.Sc. degree in physics from McMaster University, Hamilton, ON, Canada, in 1995.

He joined the Technology Access and Applications Department of Nortel Technology, Ottawa, ON, Canada in 1995, where he has been involved in the modeling and characterization of silicon bipolar transistors and integrated passive components. His current interests are in the implementation of physical and scalable inductor and transmission line models as well as the accurate extraction of distributed layout parasitics for silicon-based MMIC design.

Michael Schroter (M'93) received the Dipl.-Ing. and Dr.-Ing. degrees in electrical engineering and the "venia legendi" in semiconductor devices in 1982, 1988, and 1994, respectively, from Ruhr-University Bochum, Germany.

From 1983 to 1993, he was with the Semiconductor Devices and Integrated Circuits Group at the Ruhr-University Bochum where his main activities included compact modeling and parameter extraction for high-speed bipolar transistors and development of a mixed-mode three-dimensional device simulator as well as a CAD tool for predictive circuit design and optimization. The work was financially supported by the German Research Society, Telefunken microelectronic (TEMIC), and Siemens. From 1993 to 1996, he was with Northern Telecom and BNR, Ottawa, ON, Canada, first as a Senior Member of Scientific Staff, and later as Team Leader and Advisor. He was responsible for bipolar transistor modeling, simulation, and parameter extraction. Also, in 1994, he was appointed Adjunct Research Professor at the Carleton University, Ottawa, where he gave a graduate course on bipolar transistor modelling for integrated circuit design in 1995 and 1996. In 1996, he joined Rockwell Semiconductor Systems, Newport Beach, CA, as a Group Leader. He has been responsible for establishing the RF Device Modelling Group which is responsible for developing compact models for semiconductor devices and related parameter extraction methodologies, including both bipolar and CMOS high-speed/high-frequency applications.

Since 1994, Dr. Schroter has served on the technical program committee of the BCTM. In 1989, he received an industrially funded award for his dissertation.

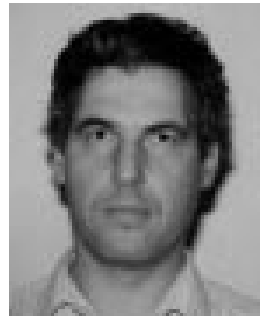

Peter Schvan (M'89) was born in Budapest, Hungary, in 1952. He received the M.S. degree in physics from Eotvos Lorand University, Budapest, in 1975 and the Ph.D. degree in electrical engineering from Carleton University, Ottawa, ON, Canada, in 1985 .

In 1985 he joined Nortel, Ottawa, where he has been working in the area of semiconductor device characterization, modeling, development of BiCMOS and bipolar technologies, and high-speed circuit design. He is currently Manager of a group responsible for evaluating various high-performance technologies and demonstrating advanced circuit concepts required for high-speed communication systems.

David L. Harame (S'77-M'83) was born in Pocatello, ID, in 1948. He received the B.A. degree in zoology from the University of California, Berkeley, in 1971 and the M.S. degree in zoology from Duke University, Durham, NC, in 1973. He received the M.S. degree in electrical engineering from San Jose State University, San Jose, CA, in 1976, and the M.S. degree in materials science and the Ph.D. degree in electrical engineering, both from Stanford University, Stanford, CA, in 1986.

In 1986 he joined the Bipolar Technology Group at the IBM T.J. Watson Research Center, Yorktown Heights, NY, where he worked on a variety of projects related to fabrication and modeling of bipolar and BiCMOS integrated circuits. The primary focus of his work was the development of SiGe-base heterojunction bipolar and BiCMOS technology. Since 1994 he has been a Senior Engineer Manager of the SiGe Technology Group at the IBM Advanced Semiconductor Technology Center, Hopewell Junction, NY. In 1997, he moved to the IBM Essex Junction, VT, manufacturing site where he is a Senior Engineer Manager of the SiGe Manufacturing Product Engineering and Development group in IBM's Microelectronic Division. His interests include Silicon and SiGe-channel FET transistors, NPN and PNP Bipolar Junction Transistors, NPN and PNP SiGe base heterojunction bipolar transistors, complementary bipolar technology, and BiCMOS technology. $\mathrm{He}$ has authored or coauthored over 100 publications. He is currently a member of the Bipolar/BiCMOS Technology Meeting Device Physics Subcommittee. 